Francesca Adam, Stefanie Föbker, Daniela Imani, Carmella Pfaffenbach, Günther Weiss, Claus-Christian Wiegandt*

\title{
Akteurslogiken bei kommunalen Integrationspolitiken für Geflüchtete in nordrhein-westfälischen Groß- und Kleinstädten
}

\section{Actors' logics concerning municipal integration policies for refugees in North Rhine-Westphalian cities and small towns}

https://doi.org/10.2478/rara-2019-0040

Eingegangen: 5. Juni 2018; Angenommen: 23. Mai 2019

Kurzfassung: Mit dem deutlichen Anstieg der Zuwanderung Geflüchteter seit Mitte der 2010er-Jahre stehen die Städte und Gemeinden mit ihrer kommunalen Ausländer- und Migrationspolitik vor neuen Herausforderungen der gesellschaftlichen Integration. Die Bewältigung dieser Herausforderungen erfolgt im Zusammenspiel verschiedener, zum Teil neuer lokaler Akteure aus dem öffentlichen (z. B. kommunale Verwaltung, Wohlfahrtsorganisationen), privatwirtschaftlichen (z. B. Arbeitgeber) und zivilgesellschaftlichen Bereich (z. B. ehrenamtliche Initiativen). In diesem Beitrag werden die verschiedenen Logiken zentraler, am Integrationsprozess beteiligter Akteure beleuchtet, um aufzuzeigen, wer unter welchen Voraussetzungen die Integrationsbedingungen vor Ort in welcher Weise gestaltet und steuert. Die Untersuchung wurde in zwei unterschiedlichen lokalen Kontexten, der Stadt Köln und dem Kreis Heinsberg, mit dem Ziel durchgeführt, Unterschiede und Gemeinsamkeiten lokaler Governance aufzuzeigen. Die Ergebnisse beruhen auf 29 Experteninterviews mit Vertretern der Stadt- und Kreisverwaltungen, der Stadtgesellschaften und der Landesebene, die im Rahmen beruflicher oder ehrenamtlicher Tätigkeit am Integrationsprozess Geflüchteter beteiligt sind. Im Ergebnis zeigen sich bei einigen Maßnahmen der Integrationspolitik verschiedene Handlungslogiken. Dies trifft erstens für das Verhältnis zwischen staatlichen Akteuren der Bundes- und Landesebene und kommunalen Akteuren zu. Dies trifft zweitens aber auch auf das Verhältnis zwischen kommunalen und zivilgesellschaftlichen Akteuren zu. Dabei spielen die räumlichen und situativen Kontexte eine differenzierende Rolle.

\section{Schlüsselwörter: Geflüchtete, kommunale Integrationspolitik, Governance, Köln, Kreis Heinsberg}

Abstract: With the significant increase in immigration of refugees since the mid-2010s, cities and municipalities are facing new challenges of social integration with their local policies on foreigners and migration. These challenges are

\footnotetext{
*Corresponding author: Prof. Dr. Claus-Christian Wiegandt, Rheinische Friedrich-Wilhelms-Universität Bonn, Geographisches Institut, Meckenheimer Allee 166, 53115 Bonn, Deutschland, E-mail: wiegandt@geographie.uni-bonn.de

Francesca Adam, Prof. Dr. Carmella Pfaffenbach, Rheinisch-Westfälische Technische Hochschule Aachen, Lehr- und Forschungsgebiet Kulturgeographie, Templergraben 55, 52056 Aachen, Deutschland

Dr. Stefanie Föbker, Daniela Imani, Rheinische Friedrich-Wilhelms-Universität Bonn, Geographisches Institut, Meckenheimer Allee 166, 53115 Bonn, Deutschland

PD Dr. Günther Weiss, Universität zu Köln, Institut für Geographiedidaktik, Gronewaldstraße 2, 50931 Köln, Deutschland
} 
handled through the interaction of various, in part new, local actors from the public sector (e.g. municipal administration, welfare organisations), the commercial sector (e.g. employers) and the civil society (e.g. voluntary initiatives). In this paper, the different logics of central actors involved in the integration processes are examined in order to show who creates and controls the local integration conditions under which preconditions. The study was conducted in two different local contexts, the city of Cologne and the district of Heinsberg, with the aim of highlighting differences and similarities in local governance. The results are based on 29 expert interviews with representatives of the city administrations, the civil society and experts of the state level who are involved in the integration process of refugees due to their professional or voluntary work. Different logics can be identified. Firstly, this applies to the relationship between state actors at the federal and state levels and local actors. Secondly, this also applies to the relationship between municipal and civil society actors. The spatial and situational contexts play a differentiating role.

Keywords: Refugees, Municipal integration policies, Governance, Cologne, District of Heinsberg

Anfangs haben wir es einfach gemacht, heute brauchen wir ein Gesundheitszeugnis für das Schneiden von Zwiebeln, da verliert man die Motivation (Aussage einer ehrenamtlichen Helferin)

\section{Einführung: Neue Herausfor- derungen für eine kommunale Integrationspolitik}

Seit Mitte der 2010er-Jahre ist Deutschland verstärkt das Ziel einer Zuwanderung von Geflüchteten, die in der Folge politischer Auseinandersetzungen und kriegerischer Konflikte im Nahen Osten, Afrika und Zentralasien sowie wirtschaftlicher Probleme in einigen Ländern ihre Heimat verlassen haben. Mit der gewachsenen Zahl an Geflüchteten stehen die Städte und Gemeinden mit ihrer kommunalen Ausländer- und Migrationspolitik vor neuen Herausforderungen der gesellschaftlichen Integration. Seit den 1960er-Jahren war die Ausländer- und Migrationspolitik gegenüber den Arbeitsmigranten zunächst als eine reine „Ausländerbeschäftigungspolitik“ und gegenüber Aussiedlern und Spätaussiedlern als eine "ethnische Inklusionspolitik" ausgerichtet. Gegenüber Asylbewerbern und geduldeten Flüchtlingen war die Politik auf Bundes- und Landesebene lange Zeit als eine "Abschottungspolitik zur Abwehr des Imports von Armut" gekennzeichnet (Filsinger 2018: 317), während auf kommunaler Ebene schleichend Veränderungen in der Integrationspolitik zu beobachten waren (Aumüller 2018: 174, 182; Filsinger 2018: 317). Die kommunale Integrationspolitik hat sich zu einem „Handlungsfeld mit eigenem Gewicht und zunehmend professioneller Fundierung" (Gesemann/Roth 2018b: 2) entwickelt. Den Städten und Gemeinden kommt damit eine „Schlüsselrolle für die Integration von Migranten“ (Bommes 2018: 100) zu, ohne die eine gesamtstaatliche Ausländer- und Migrationspolitik des Bundes und der Länder gar nicht mehr denkbar wäre.

In der jüngeren Zeit hat die kommunale Integrationspolitik auch in der Wissenschaft an Aufmerksamkeit gewonnen (Krummacher 2017; Schammann 2017a; Gesemann/Roth 2018a). Im Zuge dessen wird auch die Integration von Geflüchteten in den Städten und Gemeinden in verschiedenen Handlungsfeldern thematisiert (ElMafaalani/Weitzel/Waleciak 2016; Bauer 2017; Hinger/ Schäfer 2017; Nuissl/Domann/Engel 2019). Lange Zeit wurde dieses Thema empirisch jedoch kaum behandelt. Bis auf die ausführliche Studie von Gesemann, Roth und Aumüller (2012), eine Befragung des Bundesinstituts für Bau-, Stadt- und Raumforschung (BBSR 2008) und einige Vergleiche ausgewählter Städte im Rahmen konzeptioneller Überlegungen (Schammann 2017a) fehlt es an Studien zu den kommunalen Politikprozessen und der konkreten Ausgestaltung der lokalen Politiken mit inren Institutionalisierungen und Programmen (Aumüller 2018: 174; Bommes 2018: 104; Filsinger 2018: 317, 334).

An diesem Defizit setzt unser Beitrag an. Wir streben allerdings weder eine umfassende Politikfeldanalyse zu lokalen Akteurskonstellationen noch eine politikwissenschaftliche Implementationsforschung (vgl. Wollmann 1980; Czaika/de Haas 2013) zur Umsetzung europäischer oder nationalstaatlicher Regelungen auf die kommunale Ebene an (vgl. zu beiden Perspektiven Schammann 2017a: 95 ff.). Vielmehr wollen wir den Umgang verschiedener lokaler Akteure mit den Herausforderungen der Zuwanderung von Geflüchteten für zwei verschiedene räumliche und situative Kontexte vergleichend betrachten. Das Spektrum der lokalen Akteure umfasst dabei sowohl Personen, die sich aus beruflichen Gründen um die Integration der Geflüchteten kümmern, als auch ehrenamtlich tätige Bürger, die mit dem Anstieg der Geflüchtetenzahlen als zivilgesellschaftliche Akteure 
Verantwortung im Altagsleben der Geflüchteten vor Ort übernommen haben und damit einen wichtigen Beitrag zur Integration leisten.

Ziel unseres Beitrages ist es, in den beiden unterschiedlichen Kontexten die verschiedenen Logiken der am Integrationsprozess beteiligten lokalen Akteure zu untersuchen. Dabei baut unser Beitrag auf einer Bestandsaufnahme der kommunalen Integrationspolitik in Deutschland auf (Kapitel 2). Dadurch geraten Akteure in den Blick, die sich aktiv an der Integration der Geflüchteten vor Ort beteiligen. Uns interessiert, wer unter welchen Voraussetzungen die Integration vor Ort in welcher Weise gestaltet und steuert. Im dritten Kapitel stellen wir dazu unser methodisches Vorgehen vor. In den Ergebnissen werden wir dann im vierten Kapitel das Verhältnis der handelnden Akteure zueinander in den beiden unterschiedlichen räumlichen Kontexten vergleichend behandeln und Reibungsverluste, aber auch Synergien zwischen den Akteuren aufzeigen. Es geht uns im Kern unseres Beitrages um die lokale Governance der Integrationsprozesse von Geflüchteten seit der Phase des Ankommens.

Einen besonderen Fokus richten wir auf die lokalspezifischen Bedingungen für die verschiedenen Lebensbereiche, die für eine Integration maßgeblich sind. So treffen die Geflüchteten im Wohnungs- und Arbeitsmarkt sowie bei der Bildung auf unterschiedliche räumliche Strukturen in Groß- und Kleinstädten. Wir prüfen mit unserer Empirie, inwieweit die Integration dabei durch groß- oder kleinstädtische Zusammenhänge bzw. unterschiedliche Phasen der Zuwanderung beeinflusst wird. Im Fazit fassen wir schließlich Unterschiede und Gemeinsamkeiten zwischen den räumlichen und situativen Kontexten zusammen.

\section{Konzeptionelle Rahmung: Das Zusammenspiel verschiede- ner Akteure in der kommunalen Integrationspolitik}

In dem weiten Feld der Migrations- und Integrationsforschung beschäftigen wir uns mir der lokalen Integrationspolitik (vgl. u. a. Gesemann/Roth/Aumüller 2012; Krummacher 2017; Schammann 2017a; Bommes 2018; Filsinger 2018). Dazu werden wir im Folgenden auf das politische Mehrebenensystem in Deutschland (Kapitel 2.1) und die kommunale Integrationspolitik (Kapitel 2.2) eingehen.

\subsection{Integrationspolitik im politischen Mehrebenensystem in Deutschland}

Im föderativen System der Bundesrepublik Deutschland sind Maßnahmen zur Migration und Integration im politischen Mehrebenensystem von Bund, Ländern und Kommunen auf verschiedenen Ebenen angesiedelt (Schammann/Kühn 2016: 6 ff.; Schammann 2017a: 94). In dieser nationalen Binnenstruktur tragen vom Grundsatz her Bund und Länder die Verantwortung für die Gesetzgebung und die Förderungen in zentralen Integrationsbereichen, während Städte und Gemeinden im Bereich der kommunalen Selbstverwaltung die Integrationsförderung vor Ort gestalten und moderieren (Krummacher 2017: 83).

Im Verhältnis dieser verschiedenen politischen Ebenen wird in der internationalen Migrations- und Integrationsforschung heute ein sogenannter local turn beschrieben, das heißt, dass die kommunale Ebene eine größere Bedeutung als Handelnder (Akteur) in der Integrationspolitik erlangt hat (Penninx/Garcés-Mascareñas 2016; vgl. aber Emilsson 2015). Dieser local turn wirkt in zwei Richtungen: Auf einer horizontalen Ebene beziehen politische Entscheidungsträger zivilgesellschaftliche Akteure stärker in ihre Aktivität zur Integration ein, auf einer vertikalen Ebene ist die lokale Aktivität in komplexer Weise mit der staatlichen Aktivität verschränkt (Zapata-Barrero/Caponio/Scholten 2017: 243 f.). Dies korrespondiert mit allgemeinen Veränderungen bei der Steuerung gesellschaftlicher Prozesse zwischen dem öffentlichen, privatwirtschaftlichen und zivilgesellschaftlichen Sektor, die für die Stadtentwicklung und Stadtpolitik seit einiger Zeit unter dem Stichwort der „Urban Governance" oder der "Local Governance" behandelt werden (Einig/Grabher/lbert et al. 2005: II). Diese Prozesse zeichnen sich dadurch aus, dass sich staatliche Akteure häufig nur noch als Anstoßgeber oder Moderatoren, teils sogar nur noch als gleichberechtigte Teilnehmer in Aushandlungsprozessen verstehen. Die gesellschaftliche Steuerung wird um den Bereich der privaten Wirtschaft und der Zivilgesellschaft erweitert, um Kommunen von bestimmten Aufgaben und Kosten zu entlasten. Dadurch wird der politische Raum demokratischer Einflussnahme neu definiert (Dahme/Wohlfahrt 2018: 89).

Bei der Integration von Geflüchteten nehmen das Ehrenamt und das zivilgesellschaftliche Engagement eine besondere Rolle ein (Han-Broich 2012), aber auch in der Privatwirtschaft entstehen neue Initiativen. Die hohen Anforderungen an die kommunalen Infrastrukturen infolge der nicht vorhersehbaren Zuwanderungen haben mancherorts zu neuen Governancestrukturen und 
Akteursnetzwerken geführt (Pott/Schmiz 2018: 5), die im Weiteren im Fokus dieses Beitrags stehen. In der Folge werden Bürger und Zivilgesellschaft stärker aktiviert, sich an der Bearbeitung gesellschaftlicher Probleme zu beteiligen. Die Übernahme gesellschaftlicher Verantwortung durch den Einzelnen kann dabei eine entlastende Wirkung für den „überforderten Wohlfahrtsstaat" haben (Dahme/Wohlfahrt 2018: 80).

\subsection{Kommunale Aufgaben einer Integrationspolitik}

Schon vor dem Anstieg der Geflüchtetenzahlen im Jahr 2015 haben die Städte und Gemeinden sowie die Landkreise die kommunale Integrationspolitik als ein zentrales Thema ihrer Arbeit bewertet, das sich von einer „kommunalen Nischenrolle zu einer zentralen Zukunftsaufgabe" (Gesemann/Roth/Aumüller 2012: 13) entwickelt hat. Kommunale Integrationspolitik ist durch eine große Vielfalt an Akteuren und Aktionen in unterschiedlichen Handlungsfeldern gekennzeichnet (vgl. Bommes 2018). Dies ist bei rund 11.500 Gemeinden in Deutschland und der verfassungsmäßig garantierten kommunalen Selbstverwaltung, die gewisse Spielräume für die Ausgestaltung einer kommunalen Politik eröffnet, nicht überraschend und ist gleichzeitig Ansatzpunkt für unser Erkenntnisinteresse, die Integrationsbedingungen für Geflüchtete in Städten und Gemeinden unterschiedlicher Größenordnung zu untersuchen.

Für die Aufnahme und Integration von Geflüchteten gibt es in Deutschland bei den öffentlichen Einrichtungen unterschiedliche Zuständigkeiten auf Bundes-, Landes- und kommunaler Ebene. Auf Bundesebene sind Akteure wie das Bundesamt für Migration und Flüchtlinge (BAMF) für die Asylverfahren im Rahmen des Asylgesetzes zuständig (Schammann/Kühn 2016: 6), auf Landesebene Akteure wie Bezirksregierungen für die Unterbringung der Asylsuchenden sowie die Gewährung existenzsichernder Leistungen (Aumüller 2018: 179). Bei den aufenthaltsrechtlichen Angelegenheiten bedienen sich die Länder der kommunalen Ausländerbehörden, die hier weisungsgebundene Pflichtaufgaben wahrzunehmen haben. Dies gilt auch für die Gewährung sozialer Leistungen nach dem Asylbewerberleistungsgesetz. Städte und Gemeinden unterscheiden sich allerdings in ihrer jeweiligen Praxis, zumal hier weisungsgebundene Pflichtaufgaben mit den pflichtigen oder auch freiwilligen Selbstverwaltungsaufgaben der Kommunen verschwimmen (Schammann/Kühn 2016: 7 ff.; Schammann 2017a: 105).
Auf der kommunalen Ebene findet die Integration von Geflüchteten bei einer Vielfalt an Akteuren in unterschiedlichen Handlungsfeldern statt, die von Sprache und Bildung über Wohnen bis zu Arbeit und Beruf reichen - um die wichtigsten Felder kommunaler Integrationspolitik zu benennen (Aumüller 2018: 183). Nicht alle Handlungsfelder können in diesem Beitrag vertieft behandelt werden. Bei den Zuständigkeiten auf kommunaler Ebene ist zudem zu unterscheiden zwischen kreisfreien und meist kleineren kreisangehörigen Städten, in denen einzelne Aufgaben der kommunalen Verwaltung von den Landkreisen übernommen werden.

Mit der Verteilung der Geflüchteten auf die Kommunen beginnt ihre Integration vor Ort. Im Handlungsfeld Sprache und Bildung bilden deutsche Sprachkenntnisse einen Schlüssel für eine gelungene Integration (Aumüller 2018: 189). Zum Erwerb der deutschen Sprache und Kenntnis über deutsche Kultur sind die Integrationskurse erste Maßnahmen der Integration, die das BAMF zu verantworten hat, und die von den örtlichen Volkshochschulen oder anderen Bildungseinrichtungen durchgeführt werden (Schammann/Kühn 2016: 22). Die Kommunen sind zudem verantwortlich, frühkindliche Bildung bereitzustellen. Im schulischen Bereich liegen die meisten Aufgaben in der Zuständigkeit der Länder. Die Kommunen sind hier als Schulträger für die räumliche und sachliche Ausstattung gefordert (Massumi/von Dewitz/Grießbach et al. 2015: 41).

Im Handlungsfeld Wohnen werden die Geflüchteten zunächst nach dem Königsteiner Schlüssel auf die Länder und aus den dortigen Erstaufnahmeeinrichtungen dann durch unterschiedliche Verfahren auf die Kommunen weiterverteilt. Im Rahmen der sogenannten Anschlussunterbringung sind die Kommunen verpflichtet, Wohnraum für die Geflüchteten bereitzustellen (BBSR 2017: 18). Dies geschieht entweder in zentralen Gemeinschaftsunterkünften oder dezentral in Einzelwohnungen bzw. kleinen Wohneinheiten vor Ort (Hinger/ Schäfer 2017), wobei diese zweite Möglichkeit allerdings in Regionen mit angespannten Wohnungsmärkten beschränkt ist (Ottersbach/Wiedemann 2017: 24). In einigen Städten sollen kommunale Leitlinien helfen, die Unterbringung der Geflüchteten zielgerichtet zu steuern (Aumüller 2018: 186). Mit der Anerkennung eines Schutzstatus werden die Geflüchteten in den Grenzen der Wohnsitzauflage zu Nachfragern zumeist im preisgünstigen Segment des Mietwohnungsmarktes. Dadurch wird die kommunale Wohnungsbaupolitik aktuell noch stärker gefordert, preisgünstigen Wohnraum zu schaffen (BBSR 2017: 77). Um eine räumliche Konzentration der Geflüchteten in einzelnen Städten und Gemeinden zu vermei- 
den, hat das Land Nordrhein-Westfalen infolge des Integrationsgesetzes eine temporäre Wohnsitzauflage für anerkannte Flüchtlinge im Flüchtlingsaufnahmegesetz eingeführt, um die landesweite Verteilung zu steuern (Wiegandt 2017: 309). Danach werden die Geflüchteten für die Dauer von drei Jahren verpflichtet, ihren Lebensmittelpunkt an dem ursprünglich zugewiesenen Ort zu wählen. Dies gilt nicht für Personen, die in einem Beschäftigungs-, Ausbildungs- oder Studienverhältnis stehen, was deutlich werden lässt, dass das Leistungsprinzip in der deutschen Migrations- und Integrationspolitik an Bedeutung gewinnt (Schammann 2017b: 751).

Das Handlungsfeld Arbeit und Beruf ist ein weiterer wichtiger Baustein für die Integration. Für die Aufnahme einer Erwerbstätigkeit ist der Aufenthaltsstatus der Geflüchteten ausschlaggebend. ${ }^{1}$ In Abhängigkeit der vielfältigen Aufenthaltstitel liegt es in der Verantwortung der kommunalen Ausländerbehörden, die Ausübung einer Beschäftigung zu genehmigen. Bei dem anschließenden Bemühen, die Geflüchteten in den Arbeitsmarkt zu integrieren, übernehmen dann die Arbeitsagenturen, die Jobcenter bzw. die „Integration Points“ wichtige Aufgaben im Zusammenspiel der unterschiedlichen Akteure (Schammann/Kühn 2016: 25). Mittlerweile ist der Besuch der hierfür notwendigen Maßnahmen (z. B. Unterstützung beim Anerkennungsverfahren, berufsbezogene Sprachkurse) infolge von Veränderungen der Asylgesetze und der Beschäftigungsverordnung nicht nur anerkannten Flüchtlingen, sondern auch Asylsuchenden mit hoher Bleibeperspektive möglich. Hier zeigt sich ein bemerkenswerter Wechsel von einer Politik, die vormals auf Abschottung Geflüchteter vom deutschen Arbeitsmarkt ausgerichtet war, hin zu einer möglichst frühen Arbeitsmarktintegration ausgewählter Geflüchteter, um ihr Wissen, ihre Kreativität und ihre Arbeitskraft zu nutzen (vgl. auch Schammann 2017b: 749; Etzold 2018: 319). Allerdings ist diese Öffnung des Arbeitsmarktes in erster Linie auf die derzeitige Wirtschaftslage und den Fachkräftemangel zurückzuführen. Es bleibt offen, ob diese Haltung bei einer sich verschlechternden Wirtschaftslage aufrechterhalten wird.

Neben den vielfältigen Maßnahmen der städtischen Verwaltungen in den verschiedenen Handlungsfeldern einer kommunalen Integrationspolitik werden das Ankommen und die Integration der Geflüchteten von zivilgesellschaftlichen Akteuren (Vereine, Migrantenselbstorganisationen) unterstützt (Speth/Becker 2016: 11). Mit dem starken Anstieg der Zahl an Geflüchteten

1 www.bamf.de/FAQ-Arbeitsmarktzugang-gefluechtete-Menschen (22.03.2019). im Jahr 2015 haben zunächst ehrenamtlich viele Freiwillige den Geflüchteten schnell und unbürokratisch Hilfe geleistet und damit die seinerzeit überforderten Behörden entlastet (Fleischmann 2016). Wohlfahrtsverbände betreuen zudem Geflüchtete im Auftrag der Städte. Im Integrationsprozess können engagierte Bürger „Brücken zur Aufnahmegesellschaft bauen“ und „als Vermittler zwischen einheimischen und geflüchteten Menschen" wirken (Fleischmann 2016: o.S.).

\section{Methodisches Vorgehen: Eine Analyse zweier lokaler Kontexte in Nordrhein-Westfalen}

Um die Handlungslogiken in den kommunalen Integrationspolitiken aufzuzeigen, haben wir eine vergleichende Analyse von zwei unterschiedlichen lokalen und situativen Kontexten in Nordrhein-Westfalen gewählt: den Kontext einer Großstadt auf der einen Seite und den Kontext eines Landkreises mit Klein- und Mittelstädten auf der anderen Seite. Diese Fokussierung erfolgte auf der Grundlage der Erkenntnis, dass Integrationsprozesse von Zuwanderern und Geflüchteten wesentlich durch den jeweiligen lokalen Kontext beeinflusst werden (Schammann 2017a; Aumüller/Bretl 2008: 10). Als zentrale Einflussgrößen gelten die Größe der Stadt und die Bevölkerungsstruktur (Präsenz von ethnischen Communities), die Strukturen des städtischen Arbeitsmarktes sowie der Flüchtlingsaufnahme (Aumüller/Daphi/Biesenkamp 2015: 117). Die Auswahl von zwei unterschiedlichen Kontexten und deren vergleichende Analyse zielen darauf herauszufinden, wer jeweils die Integration von Geflüchteten auf welche Weise steuert und gestaltet (Pickvance 2005: 3). Jeder Kontext wird durch eine Fallstudie repräsentiert: der großstädtische Kontext durch die Stadt Köln und der klein-/mittelstädtische Kontext durch den Kreis Heinsberg (vgl. Abbildung 1).

Köln ist mit 1,08 Millionen Einwohnern (Stand 31.12.2016) Nordrhein-Westfalens größte und Deutschlands viertgrößte Stadt. Die Stadt verfügt über eine Nettozuwanderung, die hauptsächlich durch eine Zuwanderung aus dem Ausland und eine Zuwanderung durch 18- bis 20-Jährige getragen wird. 19,3 Prozent der Einwohner Kölns haben eine ausländische Staatsbürgerschaft, weitere 18,5 Prozent sind Deutsche mit Migrationshintergrund (Stadt Köln 2017a: 7). Personen mit türkischer Staatsangehörigkeit bilden die Mehrheit der ausländischen städtischen Bevölkerung (22,5 \%). Personen aus den Hauptherkunftsländern der Geflüchteten 


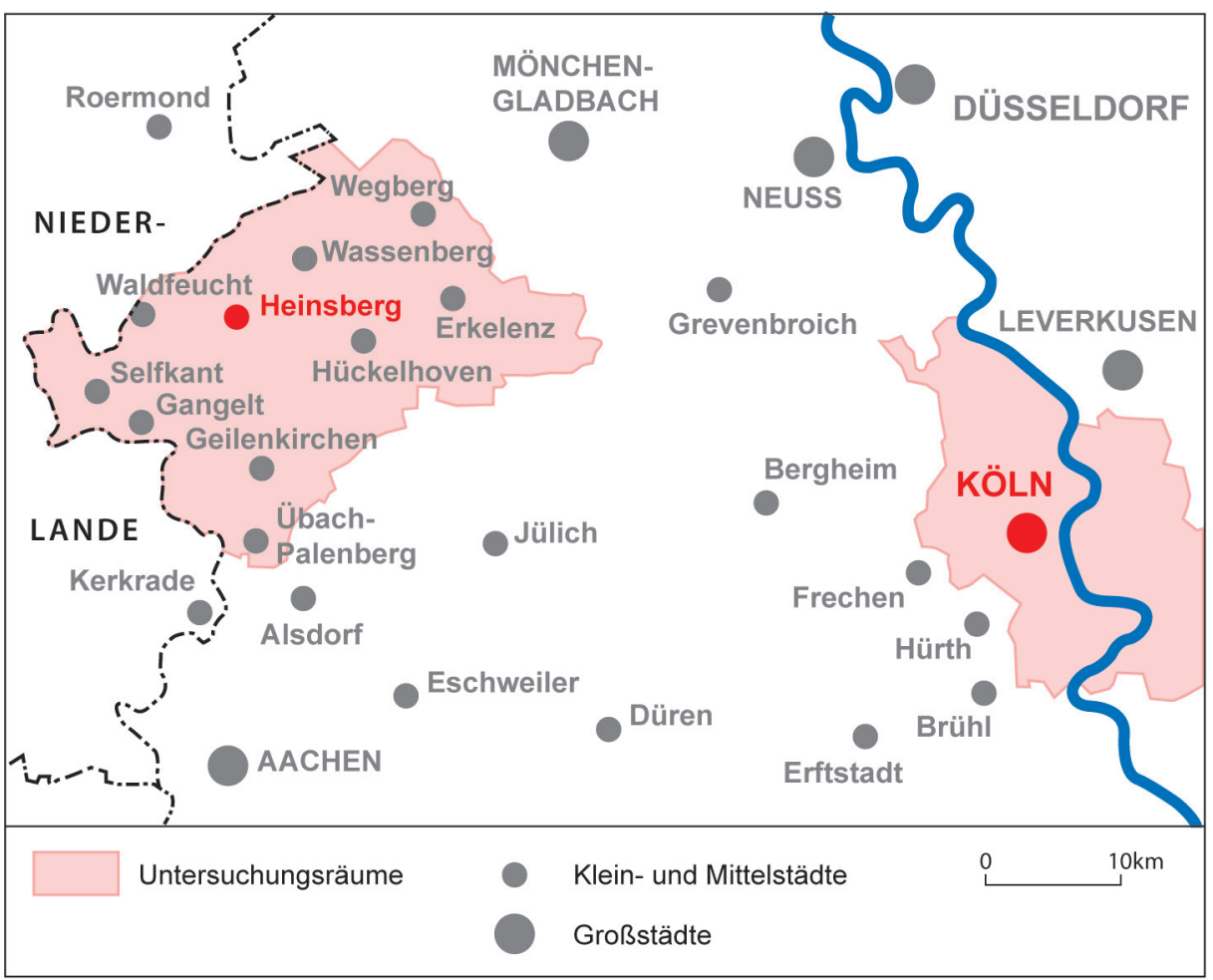

Abbildung 1: Die Untersuchungsräume Köln und Kreis Heinsberg

(Syrien, Iran und Irak) machen 6,9 Prozent aller Ausländer in Köln aus (Stadt Köln 2017b: 36). Die Stadt verfügt in vielerlei Hinsicht über einen internationalen Charakter sowie über vielfältige migrantische Infrastrukturen (Gastronomie, Vereine, religiöse Stätten), die das Bild einer vergleichsweise offenen und toleranten Stadtgesellschaft begründen und prinzipiell die Integrationsprozesse von Geflüchteten erleichtern können. Zu Beginn des Jahres 2017 lebten knapp 13.000 Geflüchtete in Unterkünften der Stadt Köln.

Der Kreis Heinsberg mit insgesamt 260.000 Einwohnern setzt sich aus zehn Kommunen in einer GröBenordnung zwischen 9.000 und 44.000 Einwohnern zusammen und ist sowohl durch gut versorgte und angebundene städtische Zentren als auch durch dünn besiedelte und peripher liegende Orte charakterisiert. Die Hälfte der Kommunen sind Mittelstädte mit mehr als 20.000 Einwohnern, während die andere Hälfte Kleinstädte mit weniger als 20.000 Einwohnern sind. Wie die Stadt Köln verfügt auch der Kreis Heinsberg über eine Nettozuwanderung, die im Jahr 2015 beachtlich angestiegen ist. 14,7 Prozent der Einwohner des Kreises besitzen eine ausländische Staatsangehörigkeit. Die Werte variieren erheblich zwischen den Kommunen und reichen von 8,4 bis zu 38,8 Prozent. Personen aus den angrenzenden Niederlanden sowie der Türkei dominie- ren dabei (Kreis Heinsberg 2017: o. S.). Zu Beginn des Jahres 2017 lebten 3.600 Geflüchtete im Kreis. Die Verteilung auf die einzelnen Städte und Gemeinden erfolgte dabei annähernd proportional zu deren Einwohnerzahl. Aufgrund der Gastarbeiteranwerbung in Zechenbetriebe des Steinkohlenbergbaus verfügen die örtlichen Gemeinschaften bereits seit vielen Jahrzehnten über Erfahrung mit der Integration von Arbeitsmigranten aus verschiedenen Ländern. Von den zehn Kommunen des Kreises haben wir fünf für das empirische Vorgehen ausgewählt: die Kreisstadt, zwei Kommunen, über die als Best-Practice-Beispiele in den Medien berichtet wurde, sowie zwei Kommunen, in denen es Spannungen zwischen der Stadtverwaltung, den Bewohnern und den Geflüchteten gab. ${ }^{2}$

Das Ziel dieses Beitrags, die verschiedenen am Integrationsprozess beteiligten lokalen Akteure und deren Logiken herauszuarbeiten und dabei Unterschiede und Gemeinsamkeiten der lokalen Governance zu identifizieren, spiegelt sich in unserem methodischen Vorgehen wider. Wir haben zwei Fallstudien verglichen, um Gemeinsamkeiten trotz unterschiedlicher Kontexte erkennen zu können (Baxter 2010: 92). Die kurzen

2 Aus Gründen des Datenschutzes werden die ausgewählten Gemeinden nicht namentlich erwähnt. 
Tabelle 1: Liste der interviewten Akteure

\begin{tabular}{|c|c|c|}
\hline & Stadt Köln & Kreis Heinsberg \\
\hline \multirow[t]{10}{*}{ Stadtverwaltung } & Kommunales Integrationszentrum Stadt Köln & Kommunales Integrationszentrum Kreis Heinsberg \\
\hline & Koordinierungsstelle für Flüchtlinge & Verwaltung von Gemeinde 1 \\
\hline & Amt für Wohnungswesen & Verwaltung von Gemeinde 2 \\
\hline & Auszugsmanagement & Verwaltung von Gemeinde 3 \\
\hline & Handwerkskammer & Verwaltung von Gemeinde 4 \\
\hline & Integration Point Köln & Integration Point Kreis Heinsberg \\
\hline & Städtische Wohnungsgesellschaft & Jobcenter Kreis Heinsberg \\
\hline & Integrationsrat & \\
\hline & Volkshochschule Stadt Köln & Volkshochschule Kreis Heinsberg \\
\hline & Grundschule in Viertel 1 & \\
\hline \multirow[t]{5}{*}{ Stadtgesellschaft } & Caritas Köln & Caritas Kreis Heinsberg \\
\hline & In Via Köln & Bistum Aachen \\
\hline & Kölner Flüchtlingsrat & \\
\hline & Bürgerinitiative in Viertel 2 & Bürgerinitiative in Gemeinde 4 \\
\hline & Bildungseinrichtung in Viertel 2 & Bürgerinitiative in Gemeinde 5 \\
\hline \multirow[t]{2}{*}{ Landesebene } & \multicolumn{2}{|l|}{ Mieterbund für Nordrhein-Westfalen } \\
\hline & \multicolumn{2}{|c|}{ Ministerium für Kinder, Familie, Flüchtlinge und Integration } \\
\hline
\end{tabular}

Beschreibungen unserer Fallstudien zeigen bereits Unterschiede und die Komplexität der Fälle, die wir untersuchen werden. Zudem haben wir ein komplexes qualitatives Vorgehen gewählt, in dem wir sowohl die Perspektiven der lokalen Akteure als auch der Geflüchteten berücksichtigen.

Zur Erfassung der Perspektive der lokalen Akteure haben wir insgesamt 29 Experteninterviews mit Vertretern der Stadtverwaltungen und der Stadtgesellschaften geführt. Die Interviewpartner haben wir aufgrund ihrer beruflichen und ehrenamtlichen Tätigkeit und Zuständigkeit im Integrationsprozess von Geflüchteten ausgewählt, wobei wir bestrebt waren, in beiden Fallstudien Experten aus ähnlichen Verwaltungsbereichen bzw. mit ähnlichen Aufgaben zu finden. Darüber hinaus haben wir Interviews mit zwei Experten geführt, die auf Landesebene mit der Unterbringung und Integration von Geflüchteten befasst sind (vgl. Tabelle 1).

In den Leitfadeninterviews mit den Experten wurden folgende Themen diskutiert: generelle Erfahrungen zur Integration von Geflüchteten im Berufsalltag, Erfahrungen im Umgang mit Geflüchteten (unter anderem Sprachprobleme und deren Lösung), Probleme, Wünsche und Bedürfnisse der Geflüchteten, die Einstellung der Bevölkerung, Erfolge und Misserfolge in der lokalen Gover- nance, diesbezügliche Verbesserungsvorschläge sowie das Integrationsverständnis der Experten. Zudem haben wir insgesamt 41 Interviews mit Geflüchteten geführt, um auch ihre Perspektive zu erfassen. In diesem Beitrag fokussieren wir jedoch ausschließlich auf die Perspektive der Stadtverwaltungen und der in den Gemeinden ehrenamtlich Tätigen (zu den Ergebnissen der Interviews mit Geflüchteten vgl. Adam/Föbker/Imani et al. 2019a; Adam/Föbker/Imani et al. 2019b).

Alle Interviews wurden mit einem Diktiergerät aufgezeichnet, anschließend transkribiert und mit einem weitgehend induktiven Kategorienschema ausgewertet. Bei der Auswertung wurde zum einen auf die Kategorien des Interviewleitfadens zurückgegriffen (wie z. B. Wohnsituation der Geflüchteten, Arbeitsmarkt, Sprachangebote und Spracherwerb, Integrationsverständnis). Zum anderen wurde induktiv ein Kategorienschema entwickelt, das die verschiedenen Aspekte der Integration aufgegriffen hat, die in den Interviews angesprochen wurden (z. B. Erfahrungen im Umgang mit Geflüchteten, Verbesserungsvorschläge zur lokalen Governance). Im empirischen Teil unseres Beitrags stehen insbesondere die Bereiche Wohnungs- und Arbeitsmarkt sowie Spracherwerb im Vordergrund. 
Zusätzlich zu den Interviews haben wir im Laufe des Forschungsprozesses drei Workshops organisiert. Auf die beiden Workshops, an denen ein Großteil der zuvor interviewten Experten der Stadt Köln, des Kreises Heinsberg sowie des Landes Nordrhein-Westfalen mitgewirkt haben, nehmen wir in diesem Beitrag Bezug. Das Ziel der Workshops war, mit den Experten die Ergebnisse des Forschungsprojektes zu diskutieren sowie einen Austausch über Erfahrungen und Meinungen anzuregen. Die als Audio-Protokoll vorliegenden und transkribierten Rückmeldungen der interviewten Experten halfen, die Ergebnisse zu validieren, unser Verständnis der lokalen Governance des Zuzugs von Geflüchteten zu erweitern und von neuen Entwicklungen in den Gemeinden Kenntnis zu erhalten.

\section{Ergebnisse: Kommunale Integrationspolitik in Köln und ausgewählten Gemeinden des Kreises Heinsberg}

Um die lokale Governance von Integrationsprozessen herauszuarbeiten, nehmen wir im Folgenden zwei Perspektiven ein. Zunächst betrachten wir die kommunale Integrationspolitik im politischen Mehrebenensystem (Kapitel 4.1). Wir zeigen, wie sich Vorgaben der Bundesund Landespolitik auf das Handeln der Akteure vor Ort auswirken, wie sie Vorgaben umsetzen oder Handlungsspielräume aktiv gestalten. Anschließend betrachten wir das Zusammenspiel kommunaler und zivilgesellschaftlicher Akteure auf der lokalen Ebene (Kapitel 4.2). Von besonderem Interesse ist dabei der Wandel der lokalen Governance in den vergangenen Jahren. Exemplarisch beziehen wir uns in der Ergebnisdarstellung auf die Handlungsfelder Arbeit, Sprache und Bildung sowie Wohnen.

\subsection{Einfluss von Vorgaben der Bundes- und Landespolitik}

Vorgaben der Bundes- und Landespolitik prägen kommunale Integrationspolitik. Ihr Einfluss - und im Gegenzug das Ausmaß der Handlungsfreiheit kommunaler Akteure - ist abhängig davon, auf welche kommunalen Aufgaben sie sich beziehen. Zwei Vorgaben, die die Integrationschancen von Geflüchteten in den letzten Jahren nicht unwesentlich mitbestimmt haben, sind das
Konzept der guten Bleibeperspektive und die Wohnsitzauflage. Sie sollen im Weiteren als Beispiele dienen, die unterschiedlichen Handlungslogiken im politischen Mehrebenensystem auf der einen Seite und in unterschiedlichen räumlichen und situativen Kontexten auf der anderen Seite zu verdeutlichen.

Das auf der Bundesebene in die Integrationspolitik eingeführte Konzept der guten Bleibeperspektive ${ }^{3}$ - das erste Beispiel - ermöglicht einen Zugang zu Integrationsmaßnahmen und einen erleichterten Arbeitsmarktzugang bereits vor der Anerkennung eines Flüchtlingsstatus. Als Asylbewerber mit guter Bleibeperspektive werden Menschen definiert, die aus Herkunftsländern mit einer Schutzquote von über 50 Prozent kommen. 2017 fielen darunter die fünf Herkunftsländer Eritrea, Irak, Iran, Syrien und Somalia. In den Handlungsfeldern Sprache und Bildung sowie Arbeitsmarktintegration wird das Prinzip angewandt bei Maßnahmen, die in der Zuständigkeit des Bundes liegen und von ihm finanziert sind. Das betrifft zum Beispiel Integrationskurse (vgl. Kapitel 2.2) und berufsvorbereitende Kurse. In den Kommunen richten sich somit unterschiedliche Akteure, wie Integration Points und Integrationskursträger, die die Maßnahmen durchführen, nach diesem Konzept.

„Der Gesetzgeber sagt, wir sollen Mittel nur für die Menschen verwenden, die auch eine Perspektive haben, hier zu bleiben. Die Menschen [aus anderen Ländern] haben sie angeblich nicht, sind aber de facto oft mehrere Jahre hier und sind oft auch sehr motiviert" (Interview 1; Verwaltung; Köln). ${ }^{4}$

Das Zitat einer Gesprächspartnerin aus Köln, die Geflüchtete in Maßnahmen vermittelt, verdeutlicht, dass das Konzept auf der lokalen Ebene zwar umgesetzt wird, gleichzeitig dort jedoch durchaus kritisch bewertet wird. Während der Bund somit für Asylbewerber aus Ländern ohne gute Bleibeperspektive keine solchen Angebote macht, nutzen Akteure auf anderen administrativen Ebenen ihre Spielräume. Das Land NordrheinWestfalen hat etwa ein Programm "Basis-Sprachkurse“ aufgelegt, das mit Mitteln aus dem Europäischen Sozialfonds gefördert wird. Die Teilnahme an diesen Kursen ist an die individuelle Bleibeperspektive von Asylbewerbern geknüpft, sodass auch Asylbewerber aus anderen Ländern daran teilnehmen dürfen. Zudem bieten auf der

3 www.bamf.de/DE/Infothek/FragenAntworten/IntegrationskurseAsylbewerber/integrationskurse-asylbewerber-node.html (22.03.2019).

4 Die Kennzeichnung der Zitate gibt Auskunft zur Herkunft der Textpassage (Interview oder Workshop), zur Akteursgruppe (Verwaltung/Zivilgesellschaft) sowie zum Untersuchungsraum. 
kommunalen Ebene ehrenamtliche Initiativen und Nichtregierungsorganisationen Sprachkurse speziell für Personen an, denen keine Integrationskurse zur Verfügung stehen. Diese Tätigkeiten werden „als wichtige Stütze für Geflüchtete" (Schammann/Kühn 2016: 23) bezeichnet und in vielen Gesprächen und unseren Workshops wertschätzend hervorgehoben. Wenn das Konzept der guten Bleibeperspektive jedoch auch die Argumentation von Akteuren in der Kommunalverwaltung prägt, wie in einer Gemeinde des Kreises Heinsberg der Fall, kann dies zu Konflikten um ehrenamtliche Sprachkurse zwischen Verwaltung und Willkommensinitiativen führen:

„Wir haben zum Beispiel auch Streit mit der Stadtverwaltung gehabt, die gesagt hat, die Ehrenamtler sollen erst [...] die Flüchtlinge [über Sprachunterricht] integrieren, wenn die eine Niederlassungserlaubnis haben. Da haben wir gesagt, nee, da fängt bei uns nicht die Integration an" (Interview 4; Zivilgesellschaft; Gemeinde im Kreis Heinsberg).

Im Handlungsfeld Arbeit belegt das Beispiel einer anderen Gemeinde im Kreis Heinsberg hingegen, wie Politik und Verwaltung auf eigene Initiative im Rahmen der kommunalen Selbstverwaltung spezifische Angebote für Menschen ohne gute Bleibeperspektive entwickeln.

"So ist auch ein tolles Projekt entstanden, was von uns initiiert worden ist, für diejenigen, die jetzt noch nicht anerkannt waren und die aber auch nicht aus Staaten mit guter Bleibeperspektive kamen [...] die konnten wir ja nur integrieren [...], indem man denen auf freiwilliger Basis, also auf Basis der Stadt oder der Integrationspartner Angebote unterbreitete. Und so ist es uns gelungen, für 30 Afghanen jeweils sechs Wochen lang einen Schreinerkurs durchzuführen, den haben wir in Zusammenhang mit der Kreishandwerkerschaft, der Handwerkskammer Aachen, dem katholischen Forum für Familien und Erwachsenenbildung und der Stadt [...] finanziert. [...] Ich hatte beim Jobcenter nachgefragt, ob es nicht auch Bundesmittel dafür gäbe, gibt's ja alles nix, eben nur für diese Top-Vier-Staaten" (Interview 6; Verwaltung; Gemeinde im Kreis Heinsberg).

Politik und Verwaltung begründen ihr Engagement ökonomisch (,Jeder, der nachher in diesen Job findet, und wenn es nur einer ist, der wiegt diese Kosten volkswirtschaftlich gesehen wieder auf" (Workshop; Verwaltung; Gemeinde im Kreis Heinsberg)) und moralisch als Verpflichtung gegenüber den der Gemeinde zugewiesenen Geflüchteten.

Letztendlich erweisen sich die Vorgaben des Bundes jedoch als sehr wirkmächtig, da sie große Gruppen von Asylbewerbern von Integrationsangeboten ausschließen. Das Angebot an alternativen Sprach- kursen und Maßnahmen zur Arbeitsmarktintegration ist vergleichsweise klein und deckt den Bedarf in den Untersuchungsgebieten nicht. Unterschiede im Umgang mit dem Konzept der guten Bleibeperspektive sind zunächst darauf zurückzuführen, wie stark die einzelnen Akteure bzw. ihre Maßnahmen durch Mittel des Bundes finanziert sind. Systematische Unterschiede zwischen Groß- und Kleinstadt sind nicht erkennbar. Vielmehr erweist sich die individuelle Einstellung von Schlüsselakteuren der kommunalen Verwaltung und Politik, aber auch einzelner Kursträger als zentral dafür, ob sie alternative Ressourcen erschließen oder sich das Konzept der guten Bleibeperspektive für ihre Argumentation zu eigen machen. Dabei mag auch der finanzielle Aspekt eine Rolle spielen. So befand sich die Gemeinde, in der der Schreinerkurs entwickelt wurde, im Gegensatz zu anderen Gemeinden im Kreis, nicht in der Haushaltssicherung. Im Konflikt um ehrenamtlich organisierte Kurse standen jedoch nicht Kosten im Mittelpunkt, sondern Einstellungsunterschiede im Umgang mit Geflüchteten (vgl. auch Schammann 2017a: 108).

Ein zweites Beispiel, wie Vorgaben der Bundesund Landespolitik die Integration Geflüchteter auf der Gemeindeebene beeinflussen, bezieht sich auf das Handlungsfeld Wohnen. Eines der zentralen Themen bei der Steuerung der Flüchtlingsintegration ist die Befürchtung, dass sich Geflüchtete räumlich konzentrieren. Durch Einführung der Wohnsitzauflage im Integrationsgesetz auf Bundesebene sowie der spezifischen Ausgestaltung im nordrhein-westfälischen Flüchtlingsaufnahmegesetz (vgl. Kapitel 2.2) soll dies verhindert werden. Den Geflüchteten wird dadurch aber untersagt, in benachbarten Gemeinden Wohnungen zu suchen, in denen die Wohnungsmärkte gegebenenfalls entspannter sind. Der angespannte Wohnungsmarkt im unteren Preissegment in Köln und Teilen des Kreises Heinsberg führt deshalb dazu, dass viele Geflüchtete nicht aus den kommunalen Unterkünften in den Mietwohnungsmarkt wechseln können.

Innerhalb der Gemeinden regelt die Wohnsitzauflage den Wohnstandort nicht. Die Möglichkeiten, dort unmittelbar Einfluss auf die Wohnungssuche der Geflüchteten zu nehmen, sind deshalb begrenzt (Noack/Raspel/ Weingarten et al. 2018: 39). In der kommunalen Steuerung der Unterbringung zeigen sich in der Folge sehr unterschiedliche Auswirkungen. In der Großstadt Köln, die durch einen äußerst angespannten Wohnungsmarkt gekennzeichnet ist, spielt die Verfügbarkeit von vorhandenen Unterkünften und Freiflächen für die Errichtung neuer Unterkünfte eine zentrale Rolle. Hier wurde insbesondere in den Jahren 2015/2016 eine intensive Debatte 
über die Standortwahl für Flüchtlingsunterkünfte geführt (vgl. Friedrichs/Leßke/Schwarzenberg 2019). Sobald die Geflüchteten diese Unterkünfte verlassen und in Mietwohnungen umziehen, regelt das knappe Angebot für preisgünstige Wohnungen die räumliche Verteilung der Geflüchteten. Zwar wird die unfreiwillige Konzentration Geflüchteter als Problem der Stadtentwicklung wahrgenommen, gleichzeitig wird die Steuerbarkeit der Verteilung aber als gering eingeschätzt. Eine Gesprächspartnerin, die Wohnungen an Geflüchtete vermittelt, formuliert in diesem Zusammenhang:

„Mir geht es erstmal darum, dass die Menschen aus dem Wohnheim rauskommen [...]. Das Problem ist auch, dass wir uns an die Mietrichtlinien des Sozialamtes und Jobcenters halten müssen. In Köln sind die Mieten sehr hoch und es gibt Stadtteile, da werden wir keine Wohnungen finden, weil unsere Mietrichtlinien weit unter dem Mietspiegel für diesen Stadtteil sind. Wir können das nicht steuern. Unser Ziel ist es, die Leute in Wohnungen zu vermitteln" (Interview 2; Verwaltung; Köln).

Eine Steuerung der räumlichen Verteilung der Geflüchteten gelingt in den kleinstädtischen Strukturen des Kreises Heinsberg eher. Inwiefern und in welche Richtung gesteuert wird, unterscheidet sich jedoch zwischen den Gemeinden deutlich. So ist die Belegungspolitik in der bereits im Kontext der Arbeitsmarktintegration stark engagierten Gemeinde (siehe oben) durch einen dezentralen Ansatz geprägt. Hier konnte die Gemeinde einen Großteil der Asylbewerber in Wohnungen unterbringen und bemüht sich darum, nach Anerkennung des Schutzstatus die Mietverträge auf die Geflüchteten zu übertragen. Darüber hinaus hat diese Gemeinde in Eigenregie in zwei verschiedenen Ortsteilen jeweils ein Mehrfamilienhaus zur Unterbringung von Geflüchteten errichtet. Diese Gemeinde argumentiert zum einen mit einer aktiven Integrationspolitik, indem Geflüchtete dezentral über das Gemeindegebiet verteilt werden, zum anderen mit der fehlenden Auslastung von Infrastrukturen. Die Neubaustandorte wurden so gewählt, dass sie zur Stabilisierung der Grundschulen in zwei Ortsteilen beitragen. Im Gegensatz dazu zeigt das Beispiel der bereits im Zusammenhang mit Konflikten zwischen Verwaltung und Ehrenamt erwähnten Gemeinde im Kreis Heinsberg, dass die Steuerung der räumlichen Verteilung auch auf eine Konzentration ausgerichtet sein kann. So wurde die dezentrale Unterbringung von Asylbewerbern mit dem Rückgang der Bewerberzahlen zugunsten einer zentralen Unterbringung in einer peripher gelegenen ehemaligen Kaserne aufgegeben.
Dieses Beispiel zeigt den deutlichen Einfluss eines Landesgesetzes mit einer gemeindescharfen Wohnsitzauflage auf die lokalen Integrationsbedingungen Geflüchteter im Handlungsfeld Wohnen. Die räumliche Verteilung innerhalb der Gemeinden ist hingegen durch das Handeln kommunaler Akteure geprägt. Hier wird ein Gegensatz zwischen Groß- und Kleinstadt deutlich bei der Steuerbarkeit von Verteilungsprozessen. Sie sind in der Großstadt geringer, was in erster Linie auf die spezifische Wohnungsmarktlage zurückzuführen ist. Das Handeln der Akteure im Kreis Heinsberg ist, analog zum ersten Beispiel des Umgangs mit der guten Bleibeperspektive, durch unterschiedliche Logiken geprägt (aktive Gestaltung der Integrationsbedingungen vs. Einschränkung der Integrationspotenziale). Von zentraler Bedeutung sind hier offenbar die Einstellungen von Schlüsselakteuren in Politik und Verwaltung.

\subsection{Zusammenspiel kommunaler und zivilgesellschaftlicher Akteure}

Seit dem Spätsommer 2015 hat sich der Kreis der Akteure, die in Städten und Gemeinden an der Steuerung der Integration Geflüchteter beteiligt sind, deutlich erweitert. Neben den kommunalen Akteuren aus Politik und Verwaltung haben insbesondere ehrenamtliche Initiativen und engagierte Privatpersonen zentrale Unterstützungsleistungen erbracht. Darüber hinaus wurden auch Akteure aktiv, die bislang nur wenige oder gar keine Berührungspunkte zu Geflüchteten hatten - etwa private Wohnungsunternehmen, Industrie- und Handelskammern, Handwerkskammern, Sparkassen, Banken, Lions- oder Rotarier-Clubs. Insgesamt sind heute mehr Akteure aus dem zivilgesellschaftlichen und privatwirtschaftlichen Bereich an der Integration Geflüchteter beteiligt als vor 2015 .

Seitdem hat die Netzwerkarbeit im Zusammenspiel der verschiedenen kommunalen, zivilgesellschaftlichen und privatwirtschaftlichen Akteure an Bedeutung zugenommen. Dies bezieht sich zum einen auf Akteure im behördlichen Kontext, die sich vor der Flüchtlingszuwanderung zum Teil kaum austauschten und deren Verhältnis mitunter durch Misstrauen und Distanz geprägt war:

„Ich kenne das vom Jobcenter normalerweise nicht, dass wir so viel Netzwerkarbeit betreiben. [...] Auch ich war viel unterwegs bei Trägern, zum Beispiel beim Deutschen Roten Kreuz, wo man einfach mal den Ablauf, wie der Integration Point und die Behörde tickt, erklären muss. Da sind sehr viele Fragezeichen und wir versu- 
chen durch unsere Arbeit auch Transparenz herzustellen. [...] Wir mussten auch viel rausgehen, um zu verstehen, was die Ausländerbehörde da macht, was das Integrationsamt, das $\mathrm{Kl}$, das Schulamt in der Sache macht und wer die Wohnheime betreibt" (Interview 1; Verwaltung; Köln).

Mit der Flüchtlingszuwanderung ist die Notwendigkeit, Integration als kommunale Querschnittsaufgabe zu verstehen (Gestring 2011: 269), sichtbarer geworden. In der Folge wurde ein stärkerer Austausch - im Kreis Heinsberg gemeindeübergreifend - initiiert. Außerdem bezieht sich die Netzwerkarbeit auf die Zusammenarbeit zwischen kommunaler Verwaltung und Zivilgesellschaft bzw. privater Wirtschaft. Insbesondere auf Seiten der Zivilgesellschaft engagierten sich in der Phase der hohen Zuweisungszahlen 2015/2016 schon früh zahlreiche bereits existierende, aber auch neu gegründete ehrenamtliche Initiativen, ohne die eine rasche Erstversorgung der Geflüchteten nicht flächendeckend hätte sichergestellt werden können. So erhielten die Kommunalverwaltungen in beiden Untersuchungsräumen anfangs private Wohnungsangebote zur Unterbringung Geflüchteter. In den meisten kleinen und mittleren Gemeinden des Kreises Heinsberg konnte durch die zahlreichen Angebote an privaten Wohnungen die Belegung von Turnhallen und Ähnlichem vermieden werden.

„ICh wurde überhäuft mit Angeboten aus der privaten Vermieterebene: Ihr könnt meine Unterkunft haben, stelle ich für Flüchtlinge zur Verfügung. Wir haben jetzt sage und schreibe, mit heutigem Stand, 78 Wohnungen gemietet. Und wir kriegen immer noch Angebote. Mir war gar nicht bewusst und präsent, dass wir so viel Leerstand hatten" (Interview 3; Verwaltung; Gemeinde im Kreis Heinsberg).

Das Zitat verweist gleichzeitig auf die besonderen Rahmenbedingungen des lokalen Wohnungsmarktes mit (zunächst) zahlreichen Leerständen, die eine frühere Unterbringung in Wohnungen ermöglichte.

Diese Vernetzung zwischen kommunalen und zivilgesellschaftlichen sowie privatwirtschaftlichen Akteuren wird von vielen Gesprächspartnern als Schlüssel für eine erfolgreiche Integrationsarbeit bezeichnet. Mittlerweile geht es hierbei weniger um die unmittelbare Versorgung und Unterbringung als vielmehr um aufenthaltsrechtliche Aspekte sowie die strukturelle Integration in den Arbeitsund Wohnungsmarkt. Nicht wenige ehrenamtlich aktive Bürger sind mittlerweile über rechtliche Belange und behördliche Verfahren gut informiert und werden von hauptamtlich Tätigen als kompetente Akteure wahrgenommen. „...als Integrationsbeauftragter nehme [ich] an deren [Initiativen von Kirchengemeinden] Sitzungen und Besprechungen teil [...]. Wir beraten diese Gremien, wobei ich sagen muss, manchmal sind diese Gremien schneller als wir, in der Kenntnis über neue Rechtsentwicklungen, wir haben zum Beispiel so einen gewissen zeitlichen Nachlauf bei gesetzlichen Reformvorhaben, wo andere etwas früher informiert sind. Leider ist das so. Das ist diese träge Bürokratie. Wir tauschen uns aus, darüber, was man besser machen kann; wir verstehen uns als die Stelle, die das Feedback aus den Hilfsorganisationen wahrnehmen möchte, so dass wir uns in unserem Handeln und Wirken auch verbessern können" (Interview 3; Verwaltung; Gemeinde im Kreis Heinsberg).

Das Zitat macht deutlich, dass die Vernetzung - hier von Verwaltung und Zivilgesellschaft - zu einem besseren gegenseitigen Verständnis, zu einem Informationsaustausch in beide Richtungen und letztlich zu einer gemeinsamen Überwindung von Hindernissen führen kann (vgl. Schiffauer 2017: 16). Dies ist wichtig in einem Feld, das durch ständige Gesetzesänderungen und eine Vielzahl an neuen Programmen gekennzeichnet ist.

Nicht immer funktioniert die Zusammenarbeit zwischen den verschiedenen Akteuren aber reibungslos. Insbesondere das Zusammenspiel zwischen ehrenamtlichen Initiativen und öffentlicher Verwaltung wird in unseren Interviews von beiden Seiten des Öfteren als schwierig bezeichnet (Schiffauer 2017: 21). Hier zeigen sich die unterschiedlichen Handlungslogiken der beteiligten Akteure. Zum einen sind die Perspektiven der verschiedenen Akteure durch unterschiedliche Zeithorizonte und Arbeitsabläufe charakterisiert. Während ehrenamtliche Initiativen schnell und flexibel agieren, wird die Verwaltung als träge wahrgenommen. Zum anderen unterscheiden sich häufig die Handlungsziele der Akteure. Das kann an Beispielen der Arbeits- und Wohnungssuche der Geflüchteten verdeutlicht werden.

Bei der Arbeitssuche befürworten ehrenamtlich tätige Bürger eher als Verwaltungsmitarbeiter eine schnelle Vermittlung auch in Hilfsjobs und ungelernte Tätigkeiten, die häufig über soziale Kontakte vermittelt werden. Damit könnte die ökonomische und psychische Situation der Geflüchteten - wenn auch eher kurzfristig - verbessert werden:

„Zwei waren bei Kronenbrot, die mussten ihren Job aufgeben, um in den Integrationskurs zu gehen (lacht). Da verzweifel' ich an solchen Sachen. Also eine bessere Integration als zu arbeiten, Kollegen zu haben, sich austauschen zu können gibt's gar nicht. Und das sind 
in beiden Fällen Familienväter, [...] die arbeiten, damit sie eine Chance haben, ihre Familie zu holen, dann holt man die da raus aus dem Arbeitsprozess" (Interview 5; Zivilgesellschaft; Gemeinde im Kreis Heinsberg).

Demgegenüber streben Vertreter der kommunalen Behörden und des Integration Point an, Geflüchtete über den Besuch von Integrationskursen und gegebenenfalls ergänzenden Maßnahmen in Ausbildungen zu bringen, um eine möglichst langfristige und stabile Arbeitsmarktintegration zu erreichen und zu verhindern, dass sie im Fall eines wirtschaftlichen Abschwungs als Ungelernte inre Arbeit verlieren und wieder von Transferleistungen abhängig werden.

Diese unterschiedlichen Handlungslogiken bei der Arbeitsvermittlung zwischen Verwaltungsmitarbeitern und Akteuren aus der Flüchtlingsberatung und dem Ehrenamt zeigen sich auch bei der Wohnungssuche:

„Zum Beispiel die Ehrenamtler, die Wohnungen vermitteln. Dann haben wir das Problem zu sagen, nein, eine $40 \mathrm{~m}^{2}$ große Wohnung für eine aus fünf Personen bestehende Familie, diesen Umzug unterstützen wir nicht. Weil wir sagen, wir haben Standards und die wollen wir auch eingehalten sehen. [...] Wir als Verwaltung verhindern das, indem wir die Mieten dann nicht übernehmen" (Workshop; Verwaltung; Köln).

„Es geht ja manchmal um die Frage des Auszugs aus der Gemeinschaftsunterkunft, wo sehr viel Stress ist und dann ist die Frage, ob eine $50 \mathrm{~m}^{2}$ große Wohnung für vier Personen nicht vielleicht besser ist als weiter in einer Turnhalle zu bleiben" (Workshop; Zivilgesellschaft; Köln).

Deutlich werden die unterschiedlichen Blickwinkel der Akteure - auf der einen Seite die Wahrung von vorgegebenen Standards, auf der anderen Seite die Lösung akuter Probleme, die vor allem durch den direkten Kontakt mit den Geflüchteten im Vordergrund stehen. Die beiden Beispiele zur Arbeits- und Wohnungssuche zeigen unterschiedliche Verständnisse der beteiligten Akteure von Integration und gelungenen Integrationsverläufen.

In der Hochphase der Zuwanderung von Geflüchteten Ende 2015 war die Zusammenarbeit zwischen den Kommunen und den zahlreichen verschiedenen, zuvor unbekannten Akteuren unterschiedlichster Professionen einerseits eine große Entlastung. Andererseits bedurfte die Steuerung und Unterstützung gerade der ehrenamtlichen Initiativen noch einmal zusätzlicher Arbeitskraft und Arbeitszeit in den öffentlichen Verwaltungen. Nicht jede Zusammenarbeit erwies sich als tragfähig oder gewinnbringend. Als Beispiel kann die zuvor erwähnte Anmietung von Wohnungen von Privatpersonen im Kreis Heinsberg genannt werden. Nicht selten befanden sich die Wohnungen in einem desolaten Zustand und mussten von den Kommunen zunächst kostenintensiv renoviert werden. Diese Wohnungen wurden mittlerweile sukzessive aufgegeben. Das folgende Zitat einer Mitarbeiterin des Kölner Integration Point verweist auf verschiedene Phasen der Netzwerkarbeit:

„Wir haben in der ersten Phase erstmal versucht zu schauen, mit welchen Netzwerkpartnern es funktioniert. Am Anfang war die Euphorie da und es wollten alle mithelfen. Jetzt muss man auch schauen, welche Netzwerke stabil funktionieren. [...] Es dauert" (Interview 1; Verwaltung; Köln).

Es wird deutlich, dass in der Netzwerkarbeit die Phase der Nachjustierung, in der die Zusammenarbeit mit einigen Akteuren aufgrund von „Unfähigkeit, Untauglichkeit, oder auch anderen [fragwürdigen] Motivationen" (Interview 3; Verwaltung; Gemeinde im Kreis Heinsberg) beendet wurde, nun von einer Phase der Konsolidierung abgelöst wird, in der die Kontakte zu stabilen Netzwerkpartnern aus dem öffentlichen, privatwirtschaftlichen und zivilgesellschaftlichen Bereich ausgebaut und verfestigt werden.

Vergleicht man das Zusammenspiel der Akteure innerhalb der Stadt Köln mit den Gemeinden des Kreises Heinsberg, zeigen sich Unterschiede, die insbesondere auf die Größe der kommunalen Verwaltungen zurückzuführen sind. Die Gesprächspartner aus dem Landkreis, die häufig allein oder in kleinen Teams in ihrem Arbeitsbereich tätig sind, bewerten insbesondere die gemeindeübergreifende Vernetzung und den damit verbundenen Austausch und Informationsgewinn als förderlich. Gleichzeitig sind die Voraussetzungen im Kreis Heinsberg für Vernetzungen besonders gut, da vielfältig auch private Kontakte bestehen und die Anzahl der Akteure überschaubar ist. In Köln wurde seitens der Gesprächspartner vor allem die mitunter schwierige Vernetzung innerhalb der Kommune thematisiert. Denn hier ist die Integrationsarbeit durch einen großen Verwaltungsapparat sowie eine schwer überschaubare Zahl weiterer Akteure geprägt. Gleichzeitig eröffnet die Größe der Verwaltung jedoch auch andere Möglichkeiten der Vernetzung. So beschreibt eine Gesprächspartnerin aus der Flüchtlingsberatung in Köln die schwierige Zusammenarbeit mit den Ausländerämtern in den Stadtbezirken und dass sie sich mit Fragen daher ausschließlich an die zentrale Ausländerbehörde der Stadt wendet. Eine Flüchtlingsinitiative in einer Gemeinde des Kreises Heinsberg steht hingegen aufgrund ihres schlechten Verhältnisses zur Verwaltungsspitze vor großen Schwierigkeiten, da in der kleinen Gemeinde keine alternativen Ansprechpartner zur Verfügung stehen. 


\section{Fazit: Kommunale Integrati- onspolitik zwischen staatlichen Vorgaben und zivilgesellschaftli- chem Engagement}

Seit dem Sommer 2015 steht die kommunale Integrationspolitik in den Städten und Gemeinden in Deutschland vor besonderen Herausforderungen, die durch die plötzliche und verstärkte Zuwanderung von Geflüchteten aus politischen Krisengebieten hervorgerufen wurden. Mit unseren eigenen empirischen Ergebnissen zur Governance der Integrationspolitik in der Millionenstadt Köln und in den kleinen und mittelgroßen Gemeinden des Kreises Heinsberg können wir die verschiedenen Handlungslogiken der am Integrationsprozess beteiligten Akteure herausarbeiten und zeigen, dass die Kommunen im Zusammenspiel der verschiedenen Akteure, die im politischen Mehrebenensystem in Deutschland zur Integration von Geflüchteten vor Ort beitragen, „unterschiedlichen Modi der Koordination“ (Einig/Grabher/lbert et al. 2005: II) unterliegen. Auch wenn das Verhältnis zwischen den Akteuren der Bundes- und Landesebene und den Kommunen nach den Erkenntnissen der Governance-Forschung in einem hierarchischen Verhältnis direkter Regelung und Kontrolle steht, zeigt unsere Empirie hier eigene lokale Interpretationsspielräume, die zu räumlichen Differenzierungen einer kommunalen Integrationspolitik führen. Verstärkt werden lokalspezifische Steuerungs- und Gestaltungsformen durch das hohe Maß der Mitwirkung zivilgesellschaftlicher Akteure bei der Integration der Geflüchteten. Das generell eher heterarchische Verhältnis zu den zivilgesellschaftlichen Akteuren, das stärker durch Kooperation und Augenhöhe geprägt ist, fördert die Vielfältigkeit kommunaler Integrationspolitiken.

Auf der einen Seite beeinflussen die Vorgaben der Bundes- und Landesbehörden die kommunalen Handlungsspielräume in den verschiedenen Aufgabenfeldern zur Unterstützung der Geflüchteten und können zu Widersprüchen und Schwierigkeiten in der kommunalen Integrationspolitik führen. Dem Bemühen zur Integration der Geflüchteten auf der kommunalen Ebene kann etwa der Anspruch einer nationalen Migrationspolitik entgegenstehen, den Geflüchteten ohne Bleibeperspektive bewusst keine Integrationsmaßnahmen in Deutschland zukommen zu lassen. Auch die Wohnsitzauflage als ein Instrument der Bundes- und Landespolitik, zu einer ausgewogenen räumlichen Verteilung der Geflüchte- ten in Nordrhein-Westfalen zu kommen, kann ihnen vor Ort den Umzug in Mietwohnungen erschweren, die in benachbarten Gemeinden zur Verfügung stehen. Die beiden Beispiele zeigen, dass Städte und Gemeinden in ihrem Handeln staatlichen Restriktionen unterliegen, die ihren eigenen Vorstellungen an eine kommunale Integrationspolitik widersprechen können.

Auf der anderen Seite sind die Städte und Gemeinden gefordert, das bemerkenswerte ehrenamtliche Engagement in der Zivilgesellschaft nicht zu gefährden. Die eingangs unseres Beitrags zitierte Aussage einer Bürgerin, die sich in der Anfangsphase der Zuwanderung spontan ehrenamtlich für die Geflüchteten eingesetzt hat, macht deutlich, dass sich einige zivilgesellschaftliche Akteure inzwischen durch kommunales Verwaltungshandeln bevormundet fühlen. Im Gegensatz zum zivilgesellschaftlichen Handeln ist das kommunale Verwaltungshandeln stark verrechtlicht und an gesetzliche Vorgaben und Standards gebunden. Für den Bereich des Wohnens konnten wir dies exemplarisch zeigen. Dadurch bilden sich gegenüber den Geflüchteten Handlungsweisen in den Kommunen heraus, die sich nicht immer mit den Vorstellungen der ehrenamtlichen Akteure decken. Die Logik des kommunalen Verwaltungshandelns, sich im Rahmen rechtlicher Vorgaben zu bewegen, passt nicht immer zu der Handlungslogik der Zivilgesellschaft, in vielen Bereichen spontan und flexibel auf neue Anforderungen zu reagieren.

Mit diesen ungelösten Widersprüchen wächst die Gefahr, dass einige zivilgesellschaftliche Akteure sich vom großen Engagement aus der ersten Phase der Ankunft der Geflüchteten verabschieden. Es droht, dass die praktizierte Willkommenskultur aus der Zeit der anfänglichen kommunalen Überforderung in behördlichen Vorgaben erstickt, die von einigen ehrenamtlich tätigen Personen als zu bürokratisch wahrgenommen werden (Han-Broich 2012: 77). Dies ist bedauerlich, weil unsere Ergebnisse zeigen, dass sich die zivilgesellschaftlichen Akteure in den vergangenen Jahren wertvolle Kompetenzen angeeignet haben. Diese Kompetenzen können im konstruktiven Zusammenspiel zwischen Verwaltung und Zivilgesellschaft die Integration von Geflüchteten nachhaltig unterstützen, wie beispielsweise beim gegenseitigen Informationsaustausch über Wohn- und Arbeitsmöglichkeiten oder veränderte rechtliche Rahmenbedingungen. Nach der Phase des Ankommens geht es deshalb im Zusammenspiel der Akteure heute darum, für Geflüchtete in einer Phase des Einlebens zu einer Balance zwischen der erforderlichen Regelung und Verrechtlichung und der individuellen 
Ausgestaltung des ehrenamtlichen Engagements und somit zu einer Etablierung stabiler, vertrauensvoller und konstruktiver Netzwerke zu kommen.

Unsere Untersuchungen waren darüber hinaus auf den Einfluss räumlich unterschiedlicher Ausgangsbedingungen für eine kommunale Integrationspolitik ausgerichtet. Hier zeigen sich zwischen der Millionenstadt Köln und den verschiedenen Gemeinden im Kreis Heinsberg sowohl Unterschiede als auch Gemeinsamkeiten im Umgang mit den Geflüchteten, die von sozialen und wirtschaftlichen Gegebenheiten vor Ort abhängig sind. Die Größe der Verwaltung in Köln hat auf der einen Seite den Nachteil, dass die Verwaltungsstrukturen mit einem hohen Maß an Arbeitsteilung für Außenstehende nur schwer nachzuvollziehen sind. Mit der stärkeren Spezialisierung ist auf der anderen Seite ein sehr differenziertes Verwaltungshandeln möglich, das auf die jeweilige Problemlage zugeschnitten ist. In den kleineren Gemeinden des Kreises Heinsberg sind die Maßnahmen zur Integration hingegen stärker an einzelne Personen gebunden, was durch eine dünne Personaldecke die Verwaltungsarbeit allerdings auch anfälliger macht. Die sehr angespannte Situation auf dem Wohnungsmarkt in Köln schränkt die Möglichkeiten der Verwaltung ein, die räumliche Verteilung der Geflüchteten zu beeinflussen. Unabhängig vom räumlichen Kontext spielen schließlich die Einstellungen und Haltungen der beteiligten Akteure gegenüber den Vorgaben der Bundes- und Landesebene bzw. gegenüber den zivilgesellschaftlichen Akteuren eine zentrale Rolle für eine gelungene Integrationspolitik.

In unserer Untersuchung haben wir uns auf zwei Fallstudien in Nordrhein-Westfalen konzentriert, die wir zwischen 2016 und 2018 bearbeitet haben. In weiteren Studien könnte es von Interesse sein, die Unterschiede in der kommunalen Integrationspolitik umfassend und systematisch in verschiedenen Städte- und Gemeindetypen zu untersuchen. Es ist zu vermuten, dass es zwischen der Situation in ost- oder westdeutschen Städten, in prosperierenden oder schrumpfenden Städten Unterschiede gibt, die von den Geflüchteten wahrgenommen werden und trotz des Instruments der Wohnsitzauflage zu weiteren Wanderungsbewegungen innerhalb Deutschlands führen werden. Zudem ist die kommunale Integrationspolitik ein überaus dynamisches Feld, das lohnt, über einen längeren Zeitraum beobachtet zu werden.

\section{Literatur}

Adam, F.; Föbker, S.; Imani, D.; Pfaffenbach, C.; Weiss, G.; Wiegandt, C.-C. (2019a): "Lost in transition"? Integration of refugees into the local housing market in Germany. In: Journal of Urban Affairs. doi: 10.1080/07352166.2018.1562302

Adam, F.; Föbker, S.; Imani, D.; Pfaffenbach, C.; Weiss, G.; Wiegandt, C.-C. (2019b): Social contacts and networks of refugees in the arrival context. Manifestations in a large city and in selected small and medium-sized towns. In: Erdkunde 73, 1. doi: 10.3112/erdkunde.2019.01.02

Aumüller, J. (2018): Die kommunale Integration von Flüchtlingen. In: Gesemann, F.; Roth, R. (Hrsg.): Handbuch Lokale Integrationspolitik. Wiesbaden, 173-198. doi: 10.1007/978-3-65813409-9

Aumüller, J.; Bretl, C. (2008): Lokale Gesellschaften und Flüchtlinge: Förderung von sozialer Integration. Die kommunale Integration von Flüchtlingen in Deutschland. Berlin.

Aumüller, J.; Daphi, P.; Biesenkamp, C. (2015): Die Aufnahme von Flüchtlingen in den Bundesländern und Kommunen. Behördliche Praxis und zivilgesellschaftliches Engagement. Stuttgart.

Bauer, I. (2017): Unterbringung von Flüchtlingen in deutschen Kommunen: Konfliktmediation und lokale Beteiligung. Osnabrück/ Bonn. = State-of-Research Papier 10, Verbundprojekt „Flucht: Forschung und Transfer".

Baxter, J. (2010): Case Studies in Qualitative Research. In: Hay, I. (Hrsg.): Qualitative research methods in human geography. Oxford, 81-97.

BBSR - Bundesinstitut für Bau-, Stadt- und Raumforschung (2008): Migration/Integration und Stadtteilpolitik. Eine ExWoSt-Studie. Bonn. = ExWoSt-Information 34/2.

BBSR - Bundesinstitut für Bau-, Stadt- und Raumforschung (Hrsg.) (2017): Integration von Flüchtlingen in den regulären Wohnungsmarkt. Bonn. = BBSR Online-Publikation 21/2017. www.bbsr.bund.de/BBSR/DE/Veroeffentlichungen/BBSROnline/2017/bbsr-online-21-2017-dl.pdf (22.03.2019).

Bommes, M. (2018): Die Rolle der Kommunen in der bundesdeutschen Migrations- und Integrationspolitik. In: Gesemann, F.; Roth, R. (Hrsg.): Handbuch Lokale Integrationspolitik. Wiesbaden, 99-123. doi: 10.1007/978-3-658-13409-9

Czaika, M.; de Haas, H. (2013): The Effectiveness of immigration policies. In: Population and Development Review 39, 3, 487508. doi: 10.1111/j.1728-4457.2013.00613.x

Dahme, H.-J.; Wohlfahrt, N. (2018): Politische Steuerung in der Stadt. In: Gesemann, F.; Roth, R. (Hrsg.): Handbuch Lokale Integrationspolitik. Wiesbaden, 75-96. doi: 10.1007/978-3-65813409-9

Einig, K.; Grabher, G.; Ibert, O.; Strubelt, W. (2005): Urban Governance. Einführung. In: Informationen zur Raumentwicklung 9/10, I-IX.

El-Mafaalani, A.; Weitzel, G.; Waleciak, J. (2016): Flüchtlinge in den Kommunen - Herausforderungen und Perspektiven. In: Stadt Land Bildung. Magazin für kommunales Bildungsmanagement 2, 6-9.

Emilsson, H. (2015): A national turn of local integration policy: multi-level governance dynamics in Denmark and Sweden. In: Comparative Migration Studies 3, 7. doi: 10.1186/s40878-0150008-5 
Etzold, B. (2018): Arbeit trotz Asyl? Erlebte Chancen und Hürden von Geflüchteten beim Zugang zu Arbeit. In: Becker, M.; Kronenberg V.; Pompe, H. (Hrsg.): Fluchtpunkt Integration. Panorama eines Problemfeldes. Wiesbaden, 319-353. doi: 10.1007/978-3-658-19430-7

Filsinger, D. (2018): Entwicklung, Konzepte und Strategien der kommunalen Integrationspolitik. In: Gesemann, F.; Roth, R. (Hrsg.): Handbuch Lokale Integrationspolitik. Wiesbaden, 315343. doi: 10.1007/978-3-658-13409-9

Fleischmann, L. (2016): Vom Flüchtling zum Mitbürger: Welchen Beitrag kann die Zivilgesellschaft in Zukunft leisten? www.bpb. de/gesellschaft/migration/kurzdossiers/228570/zivilgesellschaft-uind-integration (22.03.2019).

Friedrichs, J.; Leßke, F.; Schwarzenberg, V. (2019): Die Akzeptanz von Flüchtlingen. Eine vergleichende Studie sechs deutscher Wohngebiete. In: Raumforschung und Raumordnung | Spatial Research and Planning 77, 4. doi: 10.2478/rara-2019rara-2019-0015

Gesemann, F.; Roth, R. (Hrsg.) (2018a): Handbuch Lokale Integrationspolitik. Wiesbaden. doi: 10.1007/978-3-658-13409-9

Gesemann, F.; Roth, R. (2018b): Einleitung. In: Gesemann, F.; Roth, R. (Hrsg.): Handbuch Lokale Integrationspolitik. Wiesbaden, 1-7. doi: 10.1007/978-3-658-13409-9

Gesemann, F.; Roth, R.; Aumüller, J. (2012): Stand der kommunaIen Integrationspolitik in Deutschland. Berlin.

Gestring, N. (2011): Kommunale Konzepte zur Integration von Migranten. In: Hanesch, W. (Hrsg.): Die Zukunft der „Sozialen Stadt". Strategien gegen soziale Spaltung und Armut in den Kommunen. Wiesbaden, 257-274. doi: 10.1007/978-3-53192637-7

Han-Broich, M. (2012): Ehrenamt und Integration. Die Bedeutung sozialen Engagements in der (Flüchtlings-) Sozialarbeit. Wiesbaden. doi: 10.1007/978-3-531-18689-4

Hinger, S.; Schäfer, P. (2017): Wohnst Du schon - oder wirst Du noch untergebracht? Eine Bestandsaufnahme der Wohnsituation Asylsuchender in Deutschland. www.bpb.de/gesellschaft/ migration/kurzdossiers/243947/wohnst-du-schon-oder-wirstdu-noch-untergebracht (22.03.2019).

Kreis Heinsberg (2017): Sozialraum-Monitoring [unveröffentlichtes Manuskript]. Heinsberg.

Krummacher, M. (2017): Kommunale Integrationspolitik in Deutschland. Fakten, Entwicklungstrends, Widersprüche, Perspektiven. In: Geisen, T.; Riegel, C.; Yildiz, E. (Hrsg.): Migration, Stadt und Urbanität. Perspektiven auf die Heterogenität migrantischer Lebenswelten. Wiesbaden, 79-96. doi: 10.1007/9783-658-13779-3

Massumi, M.; von Dewitz, N.; Grießbach, J.; Terhart, H.; Wagner, K.; Hippmann, K.; Altinay, L. (2015): Neu zugewanderte Kinder und Jugendliche im deutschen Schulsystem: Bestandsaufnahme und Empfehlungen. Köln.

Noack, M.; Raspel, J.; Weingarten, J.; Wohlert, J. (2018): Unterbringung geflüchteter Menschen in Essen. Eine Frage der Verteilungsgerechtigkeit. In: Standort. Zeitschrift für Angewandte Geographie 42, 1, 35-41. doi: 10.1007/s00548-018-0524-2

Nuissl, H.; Domann, V.; Engel, S. (2019): Integration als kommunalpolitische Aufgabe. Die Erschließung eines sich neu formierenden lokalen Politikfeldes. In: Raumforschung und Raumordnung | Spatial Research and Planning 77, 4. doi: 10.2478/ rara-2019-rara-2019-0016
Ottersbach, M.; Wiedemann, P. (2017): Die Unterbringung von Flüchtlingen in nordrhein-westfälischen Kommunen. Aspekte eines integrierten Gesamtkonzepts zur nachhaltigen Integration von Flüchtlingen am Beispiel von Köln. Düsseldorf.

Penninx, R.; Garcés-Mascareñas, B. (2016): Integration Policies of European Cities in Comparative Perspective: Structural Convergence and Substantial Differentiation. In: Migracijske i etničke teme 32, 2, 155-189. doi: 10.11567/met.32.2.1

Pickvance, C. (2005): The four varieties of comparative analysis: the case of environmental regulation. Paper for Conference on Small and large-N comparative solutions, University of Sussex, 22-23 September 2005. eprints.ncrm.ac.uk/57/1/ chrispickvance.pdf (22.03.2019).

Pott, A.; Schmiz, A. (2018): Migration und Flucht als Forschungsthemen der Geographie. In: Standort. Zeitschrift für Angewandte Geographie 42, 1, 3-9. doi: 10.1007/s00548-018-0517-1

Schammann, H. (2017a): Stadt, Land, Flucht. Konzeptionelle Überlegungen zum Vergleich städtischer Flüchtlingspolitik in Deutschland. In: Barbehön, M.; Münch, S. (Hrsg.): Variationen des Städtischen - Variationen lokaler Politik. Wiesbaden, 91-117. doi: 10.1007/978-3-658-13394-8

Schammann, H. (2017b): Eine meritokratische Wende? Arbeit und Leistung als neue Strukturprinzipien der deutschen Flüchtlingspolitik. In: Sozialer Fortschritt 66, 11, 741-757. doi: 10.3790/ sfo.66.11.741

Schammann, H.; Kühn, B. (2016): Kommunale Flüchtlingspolitik in Deutschland. Bonn.

Schiffauer, W. (2017): Einleitung. Eine neue Bürgerbewegung. In: Schiffauer, W.; Eilert, A.; Rudloff, M. (Hrsg.): So schaffen wir das. Eine Zivilgesellschaft im Aufbruch. 90 wegweisende Projekte mit Geflüchteten. Bielefeld, 13-35.

Speth, R.; Becker, E. (2016): Zivilgesellschaftliche Akteure und die Betreuung geflüchteter Menschen in deutschen Kommunen. Berlin. = Opusculum 92. www.maecenata.eu/images/resources/2016 op92.pdf (22.03.2019).

Stadt Köln (2017a): Einwohnerentwicklung 2016 - Weiterhin Wachstum in Köln. Köln.

Stadt Köln (2017b): Statistisches Jahrbuch 2016. Köln.

Wiegandt, C.-C. (2017): Wohnorte von Flüchtlingen in Deutschland. Eine Balance zwischen freier Wahl und Zuweisung. In: Becker, M.; Kronenberg, V.; Pompe, H. (Hrsg.): Fluchtpunkt Integration. Panorama eines Problemfeldes. Wiesbaden, 299-318. doi: 10.1007/978-3-658-19430-7

Wollmann, H. (Hrsg.) (1980): Politik im Dickicht der Bürokratie. Beiträge zur Implementationsforschung. Opladen.

Zapata-Barrero, R.; Caponio, T.; Scholten, P. (2017): Theorizing the 'local turn' in a multi-level governance framework of analysis: A case study in immigrant policies. In: International Review of Administrative Sciences 83, 2, 241-246. doi: 10.1177/0020852316688426 